\title{
Suscetibilidade à cinetose em crianças de oito a onze anos
}

\author{
Susceptibility to motion sickness in children from eight to eleven years \\ Susceptibilidad al cinetosis en niños de 8 a 12 años de edad \\ Bruna Teixeira', Rafaela Soares Rech ${ }^{2}$, Pricila Sleifer ${ }^{3}$
}

RESUMO I A cinetose se caracteriza pela intolerância ao movimento, resultante de um conflito sensorial entre os sistemas visual, proprioceptivo e vestibular. Na população infantil, a cinetose é frequente, porém o difícil diagnóstico acaba subestimando a prevalência nesse grupo específico. As alterações vestibulares pediátricas têm importante influência no desenvolvimento infantil. 0 objetivo do artigo foi analisar a suscetibilidade à cinetose em crianças e verificar possíveis fatores associados, bem como identificar diferenças entre as respostas, quando comparados os sexos, as faixas etárias e a autopercepção dos pais. Trata-se de um estudo transversal. A amostra de conveniência consecutiva foi constituída por crianças de ambos os sexos, com idade entre oito e onze anos. Para avaliar a suscetibilidade à cinetose, foi aplicado o motion sickness questionnaire short form (MSSQ), realizado individualmente com cada criança. A análise estatística foi realizada por meio do SPSS Versão v.21. Adotou-se nível de significância de 0,05. Utilizaram-se os testes estatísticos Kolmogorov-Smirnov; T de Student; Anova e teste de Friedman. Ao total, foram analisadas 223 crianças. Observou-se que 89,7\% das crianças avaliadas eram suscetíveis à cinetose. Houve diferença significativa na comparação da suscetibilidade à cinetose entre os sexos, sendo as meninas, mais suscetíveis em relação aos meninos $(p=0,001)$. Na comparação entre as faixas etárias, não houve significância estatística. Crianças com onze anos apresentaram maior suscetibilidade à cinetose. Houve diferença nas respostas relatadas pelas crianças e pais sobre a suscetibilidade das crianças à cinetose.
Descritores | Enjoo devido ao Movimento; Equilibrio Postural; Crianças; Prevalência.

ABSTRACT | Motion sickness is characterized by intolerance to movement, resulting from a sensory conflict between the visual, proprioceptive and vestibular systems. In the child population, motion sickness is frequent, but the difficult diagnosis ends up underestimating the prevalence in this specific group. Pediatric vestibular changes are of great importance in child development. The objective was to analyze the susceptibility to motion sickness in children and to verify possible associated factors, as well as to identify differences in the responses when compared to sex, age group and parents' self-perception. This is a cross-sectional study. The consecutive convenience sample consisted of children of both sexes, aged between eight and eleven years old. The motion sickness questionnaire short form (MSSQ) was applied individually with each child. Statistical analysis was performed using the SPSS Version v.21 (Chicago: SPSS). A significance level of 0,005 was adopted. Kolmogorov-Smirnov, Student's T, Anova and Friedman's were the statistical tests used. In total, 223 children were analyzed. $89.7 \%$ of the sample was susceptible to motion sickness. There was a significant difference in the comparison of susceptibility to motion sickness between genders, with girls being more susceptible, compared to boys ( $p=0.001$ ). When comparing age groups, there was no statistical significance. Eleven-year-old children were more susceptible to motion sickness. There was a difference in the responses reported by children and parents about the children's susceptibility to motion sickness.

'Universidade Federal de Ciências da Saúde de Porto Alegre (UFCSPA) - Porto Alegre (RS), Brasil. E-mail: bruuteixeiraa@gmail.com. ORCID-0000-0001-9425-958X

${ }^{2}$ Universidade Federal de Ciências da Saúde de Porto Alegre (UFCSPA) - Porto Alegre (RS), Brasil. E-mail: rafasoaresrech@hotmail. com. ORCID-0000-0002-3207-0180

${ }^{3}$ Universidade Federal do Rio Grande do Sul (UFRGS) - Porto Alegre (RS), Brasil. E-mail: pricilasleifer@gmail.com.

ORCID-0000-0001-6694-407X 
Keywords | Motion Sickness; Postural Balance; Children; Prevalence.

RESUMEN I El cinetosis es la intolerancia al movimiento pasivo, resultado de un conflicto sensorial entre los sistemas visual, propioceptivo y vestibular. La población infantil padece con frecuencia del cinetosis, pero su prevalencia se subestima debido al difícil diagnóstico en este grupo específico. Los trastornos vestibulares en niños influyen significativamente en su desarrollo. El objetivo de este artículo fue analizar la susceptibilidad al cinetosis en niños y sus factores asociados, así como identificar las diferencias entre las respuestas en la comparación entre los sexos, los grupos de edad y la autopercepción de los padres. Se trata de un estudio transversal. La muestra de conveniencia consecutiva estuvo compuesta de niños de ambos sexos, con edades comprendidas entre 8 y 12 años. Para evaluar la susceptibilidad al cinetosis, se aplicó el motion sickness questionnaire short form (MSSQ) de manera individual en cada niño. Para el análisis estadístico se utilizó el software SPSS, versión 21.0. El nivel de significancia adoptado fue de 0,05. Se utilizaron pruebas estadísticas de KolmogorovSmirnov; T de Student; Anova y test de Friedman. Participaron en total 223 niños. Se observó que el 89,7\% de los niños evaluados eran susceptibles al cinetosis. Hubo una diferencia significativa en la comparación de la susceptibilidad al cinetosis entre los sexos, en la cual las niñas eran más susceptibles que los niños ( $p=0,001)$. En cuanto a los grupos de edad, no hubo significación estadística. Los niños de 12 años fueron más susceptibles al cinetosis. Hubo una diferencia en las respuestas informadas por los niños y los padres sobre la susceptibilidad de los niños al cinetosis.

Palabras clave | Mareo por Movimiento; Balance Postural; Niños; Prevalencia.

\section{INTRODUÇÃO}

A cinetose, também conhecida como enjoo do movimento, caracteriza-se pela intolerância ao movimento, devido ao conflito sensorial entre os sistemas visual, proprioceptivo e vestibular. Em sua grande maioria, o conjunto de sintomas associados à cinetose, como náusea, cefaleia, sudorese e vômito, resultam de estímulos de movimentos não habituais. Meios de transporte e veículos como automóveis, navios, trens, metrôs, aviões e, até mesmo, elevadores são grandes estimuladores da cinetose ${ }^{1,2}$.

Estudos apontam que a cinetose é mais frequente em crianças, manifestando-se por volta dos seis e sete anos de idade e relatam que o pico do transtorno se dá por volta dos dez anos ${ }^{1-3}$. Em relação ao sexo, segundo estudo, as mulheres são mais acometidas, sendo fatores hormonais, como menstruação e gravidez, possíveis agravantes da condição ${ }^{4}$. As causas neurofisiológicas para o acometimento da cinetose ainda são desconhecidas. Uma das hipóteses é que ela ocorra devido a um conflito sensorial, a uma imaturidade do sistema vestibular, que pode ou não estabilizar-se com o avanço da idade, à influência de fatores hormonais, como a menstruação e a gravidez, bem como ao processo de habituação ${ }^{1-4}$.

Acredita-se que, embora frequente, a prevalência da cinetose na população infantil ainda seja subestimada, o que pode ser explicado pelo difícil diagnóstico das alterações vestibulares em crianças ${ }^{3,5}$. A identificação dos sintomas é subjetiva, pois depende da autopercepção e queixa do próprio paciente. Muitas vezes, a tontura não é entendida como um sintoma anormal, fazendo com que essa população possua dificuldades para referir o desconforto ${ }^{5}$.

Além disso, a percepção dos sintomas da cinetose pelo adulto responsável e o futuro direcionamento da criança para uma avaliação clínica nem sempre acontece $e^{1,6}$. Quando percebidos, os sintomas raramente são associados às vestibulopatias. A prevalência de tonturas de origem vestibular na população infantil varia entre 7,7 e 15,0\% ${ }^{6,7}$.

A abordagem clínica das alterações vestibulares muitas vezes é vista como desnecessária. A experiência clínica, no entanto, demonstra que, assim como o adulto, a criança pode sofrer repercussões das alterações em sua vida cotidiana, apresentando comprometimento cognitivo e isolamento social que influenciam direta e negativamente em seu desenvolvimento ${ }^{8,9}$.

Percebeu-se, durante a revisão bibliográfica da literatura científica, a escassez de estudos atuais a respeito da cinetose na população infantil. Há pesquisas que abordam as alterações vestibulares de modo geral, bem como a reabilitação vestibular ${ }^{2,8,9}$. No entanto, na busca pela prevalência da cinetose em crianças e por estudos que avaliam esta alteração específica, poucos estudos foram encontrados na população infantil ${ }^{3,10-14}$.

Constatou-se a importância de se identificar precocemente os sintomas de alterações vestibulares e evitar as possíveis consequências e agravantes, como o isolamento social e as dificuldades de aprendizagem. Observando-se a relevância clínica do assunto, o objetivo do presente estudo foi avaliar a suscetibilidade à cinetose em crianças e verificar possíveis fatores associados, bem como identificar 
diferenças entre as respostas quando comparadas ao sexo, a faixa etária e a autopercepção dos pais.

\section{METODOLOGIA}

Trata-se de um estudo transversal. A amostra foi composta por crianças com idade entre oito e onze anos, estudantes do segundo ao sexto ano do ensino fundamental da rede pública de Porto Alegre e de sua região metropolitana, a partir de uma amostragem não probabilística por conveniência consecutiva. Para estimar o tamanho de efeito padronizado de 0,9 foi calculado um tamanho amostral de 192 indivíduos. Foi aceito o nível de significância de 0,05 com poder de $90 \%$ (Epilnfo - Statcal).

Incluiu-se no estudo crianças com idade entre oito e onze anos, estudantes da rede pública de ensino que não tivessem perda auditiva, nem doenças de base que ocasionassem sintomas semelhantes aos da cinetose, como sudorese, náusea e cefaleia. Foram excluídas crianças com algum comprometimento físico e/ou neurológico que inviabilizasse a aplicação do questionário e crianças que não aceitaram participar da pesquisa.

A coleta de dados iniciou-se em agosto de 2018 e encerrou-se em setembro de 2019 . O projeto de pesquisa foi apresentado e aprovado pelas instituições escolhidas e os termos de consentimento e esclarecimento assinados pelos pais/e ou responsáveis e pelas crianças.

O questionário utilizado no estudo para a avaliação da suscetibilidade à cinetose foi o motion sickness questionnaire short form (MSSQ), desenvolvido por Reason e Brand ${ }^{15}$, condensado e simplificado em sua pontuação por Golding ${ }^{16}$, e traduzido e adaptado para o português por França e Branco-Barreiro ${ }^{5}$. Aplicaram-se somente as questões relacionadas à presença de cinetose na infância (Motion Sickness A - MSA). O escore total foi obtido por meio da multiplicação da pontuação por nove, e este resultado, dividido também pelo valor de nove, subtraído o número de geradores não utilizados pela criança, ou seja: $($ escore total = pontuação $M S A \times 9)$ / (9-número de transportes não utilizados).

O instrumento é composto por nove ambientes e/ou estímulos que desencadeiam a cinetose, inclusive meios de transporte e entretenimento. São eles: "carros”, “ônibus ou vans", "trens", “aviões”, "barcos pequenos”, "navios ou balsas", "balanços em parquinhos", "gira-gira em parquinhos" e "brinquedos em parques de diversões”. Como resposta, há cinco opções disponíveis: "nunca experimentou", "nunca ficava enjoado", "raramente ficava enjoado", "às vezes ficava enjoado" e "sempre ficava enjoado". A pontuação do questionário varia de 0 a 3 , sendo 0 aplicado a "não se aplica/nunca utilizou ou "nunca ficava enjoado", 1 ponto a "raramente ficava enjoado", 2 pontos a "às vezes ficava enjoado" e 3 pontos a "sempre ficava enjoado".

A análise descritiva dos dados foi realizada. Para verificar a significância estatística da distribuição das médias do MSA realizou-se o teste de KolmogorovSmirnov. Nos casos de distribuição normal foram utilizados os testes T de Student e Anova. Em caso de rejeição da hipótese de normalidade utilizou-se o teste não paramétrico de Friedman. $O$ valor para rejeição da hipótese nula foi $\mathrm{p}<0,05$. As análises foram realizadas com auxílio do software SPSS Versão v.21.

\section{RESULTADOS}

A amostra estudada, descrita na Tabela 1, foi composta por 223 crianças, com idade entre oito e onze anos, sendo 109 do sexo feminino $(48,9 \%)$ e 114 do sexo masculino $(51,1 \%)$. Os participantes são estudantes do segundo ao sexto ano do ensino fundamental de escolas da rede pública de Porto Alegre e da região metropolitana. Apenas $12,6 \%$ da amostra apresentou queixas de equilíbrio e/ou aprendizagem. A média de idade dos participantes foi de $9,14( \pm 1,02)$ e a média do escore total no MSA foi de $7,42( \pm 5,91)$.

Tabela 1. Descrição da amostra estudada. Porto Alegre, 2019 $(n=223)$

\begin{tabular}{|c|c|}
\hline Variável & n (\%) \\
\hline \multicolumn{2}{|l|}{ Sexo } \\
\hline Feminino & $109(48,9 \%)$ \\
\hline Masculino & $114(51,1 \%)$ \\
\hline \multicolumn{2}{|l|}{ Idade } \\
\hline 8 anos & $77(34,5 \%)$ \\
\hline 9 anos & $57(25,6 \%)$ \\
\hline 10 anos & $67(30,0 \%)$ \\
\hline 11 anos & $22(9,9 \%)$ \\
\hline \multicolumn{2}{|l|}{ Escolaridade } \\
\hline $2^{\circ}$ Ano & $39(17,5 \%)$ \\
\hline 3Ano & $73(32,7 \%)$ \\
\hline $4^{\circ} \mathrm{Ano}$ & $76(34,1 \%)$ \\
\hline 5Ano & $30(13,5 \%)$ \\
\hline 6Ano & $5(2,2 \%)$ \\
\hline \multicolumn{2}{|l|}{ Queixa } \\
\hline Sem queixa & $195(87,4 \%)$ \\
\hline Dificuldade de aprendizagem & $14(6,3 \%)$ \\
\hline Dificuldade de equilíbrio & $14(6,3 \%)$ \\
\hline
\end{tabular}


A prevalência da suscetibilidade à cinetose na infância (MSA) encontrada na amostra em estudo foi de 89,7\%, o que representa 196 crianças com sintomas de cinetose. Apenas 27 crianças não referiram quaisquer sintomas e obtiveram pontuação zero, representando $12,1 \%$ da amostra.

Conforme a Tabela 2, na comparação entre os sexos, obteve-se significância estatística ( $p$-valor $<0,001$ ), na qual as meninas apresentaram um escore total médio 2,73 $(1,21 ; 4,26)$ vezes maior do que o observado em meninos.

Tabela 2. Diferença das médias do escore total do MSA, entre os sexos. Porto Alegre, $2019(n=223)$

\begin{tabular}{ccccc}
$\begin{array}{c}\text { Média MSA } \\
\text { Feminino }\end{array}$ & $\begin{array}{c}\text { Média MSA } \\
\text { Masculino }\end{array}$ & $\begin{array}{c}\text { Diferença } \\
\text { das Médias }\end{array}$ & $\begin{array}{c}\text { Intervalo de } \\
\text { Confiança } \\
95 \%\end{array}$ & P-valor* \\
8,80 & 6,06 & 2,73 & $(1,21 ; 4,26)$ & $<0,001$ \\
\hline
\end{tabular}

MSA: Motion sickness A

*Teste T de Student

Comparando-se as faixas etárias (Tabela 3), não se obteve significância estatística, no entanto, observou-se um aumento do escore médio do MSA com o avanço da idade.

Tabela 3. Comparação das faixas etárias. Porto Alegre, 2019. ( $(n=223)$

\begin{tabular}{lcccc}
\multicolumn{1}{c}{ Idade } & Média MSA & $\begin{array}{c}\text { Diferença } \\
\text { das médias }\end{array}$ & $\begin{array}{c}\text { Intervalo de } \\
\text { Confiança } \\
95 \%\end{array}$ & P-valor* \\
\hline $8-9$ anos & 8,43 & 1,27 & $(-1,42 ; 3,95)$ & 0,613 \\
$8-10$ anos & 8,93 & 0,61 & $(-1,95 ; 3,18)$ & 0,926 \\
$8-11$ anos & 8,67 & 0,47 & $(-3,24 ; 4,19)$ & 0,988 \\
$9-10$ anos & 9,54 & $-0,65$ & $(-3,42 ; 2,12)$ & 0,929 \\
$9-11$ anos & 9,56 & $-0,79$ & $(-4,65 ; 3,06)$ & 0,951 \\
$10-11$ anos & 10,25 & $-0,14$ & $(-3,92 ; 3,63)$ & 1,000
\end{tabular}

MSA: Motion Sickness A

*Análise de Variância (Anova)

$\mathrm{Na}$ tabela 4, a análise não paramétrica da comparação das medianas das respostas dos pais em relação ao resultado do MSA demonstrou diferença estatisticamente significativa $(\mathrm{p}=0,029)$.

Tabela 4. Comparação das medianas do MSA realizado com os pais e com os filhos. Porto Alegre, 2019. (n=23)

$\begin{array}{cccc}\begin{array}{c}\text { Mediana MSA } \\ \text { Filhos }\end{array} & \begin{array}{c}\text { Mediana MSA } \\ \text { Pais }\end{array} & \begin{array}{c}\text { Diferença das } \\ \text { Medianas }\end{array} & \text { P-valor* } \\ 4,00 & 3,00 & 1,00 & 0,029\end{array}$

MSA: Motion Sickness A

*Teste de Friedman

\section{DISCUSSÃO}

A cinetose caracteriza-se por uma intolerância ao movimento, manifestando-se durante a locomoção em veículos, devido a um conflito entre as informações sensoriais, vestíbulo-visuais ou intravestibulares ${ }^{10-13}$. A cinetose tem influência sobre a qualidade de vida dos indivíduos, visto o grande desconforto que proporciona. Apesar da elevada prevalência da cinetose na população mundial, a alteração ainda não é vista como doença e, na maioria das vezes, o indivíduo convive por anos antes de procurar um atendimento especializado ${ }^{3,17}$.

A prevalência da suscetibilidade à cinetose na infância neste estudo foi de $89,7 \%$, aproximando-se do percentual encontrado em um estudo similar, que obteve prevalência de $76,3 \%{ }^{2}$. Outra pesquisa, também realizada na população infantil, verificou uma prevalência menor, de $56 \%{ }^{18}$. No entanto, a presença de suscetibilidade à cinetose continuou sendo maior em relação à ausência. Os estudos analisados utilizaram o mesmo protocolo (MSSQ) para avaliar a suscetibilidade à cinetose em crianças.

Visto que a prevalência encontrada no presente estudo foi superior às encontradas na literatura científica, formularamse hipóteses para justificar esse achado. A primeira é relativa ao tamanho amostral, que foi significativamente maior neste estudo em relação aos demais. A segunda hipótese é relativa à modalidade de ensino das crianças participantes. Enquanto o presente estudo avaliou crianças da rede pública de ensino, um estudo de São Paulo realizou a pesquisa com crianças da rede privada ${ }^{1}$. Desta forma, a presença de diferenças socioculturais e econômicas pode ter sido uma variável para os achados.

No presente estudo, a suscetibilidade à cinetose foi maior em meninas (média $=8,80$ ) em relação aos meninos (média $=6,06$ ), com significância estatística $(\mathrm{p}<0,001)$. Um estudo que utilizou o mesmo protocolo de avaliação encontrou dados semelhantes, identificando maior suscetibilidade à cinetose em crianças do sexo feminino, embora sem significância estatística e com médias inferiores às encontradas neste estudo ${ }^{3}$. Em outra pesquisa ${ }^{19}$, também houve diferença estatisticamente significante na comparação entre os sexos, na qual meninas tiveram escore do MSA maior do que os meninos.

Estudos afirmam que sexo e idade são duas variáveis importantes para avaliação da suscetibilidade à cinetose. Pesquisas em transporte marítimo, terrestre e aéreo indicam que as mulheres são mais suscetíveis a enjoos do que os homens ${ }^{1,14,20}$. Tais achados corroboram os resultados encontrados no presente estudo, em que meninas pontuaram mais no MSA, obtendo-se significância estatística.

A literatura científica afirma que a suscetibilidade à cinetose incide por volta dos seis aos sete anos de idade e atinge seu pico entre os nove e dez anos ${ }^{14,19}$. O fator idade é bastante associado à suscetibilidade à cinetose, 
sendo mais comum em crianças de nove e dez anos de idade $^{3}$. No presente estudo, observou-se média do MSA maior com o avanço da idade, sendo as crianças com onze anos as mais suscetíveis à cinetose, seguidas das crianças de dez e nove anos de idade ${ }^{4,19}$.

Neste estudo, optou-se por analisar também a comparação entre as respostas relatadas pelas crianças e a percepção de seus pais e/ou responsáveis sobre a suscetibilidade à cinetose dos escolares. Não foram encontrados estudos que fizessem a mesma relação. Especula-se que a dificuldade de as crianças relatarem seus sintomas, bem como a não identificação dos pais dos sinais apresentados por seus filhos, sejam alguns dos fatores que contribuem para a dificuldade e a escassez de diagnósticos vestibulares na população infantil.

$\mathrm{Na}$ comparação destes resultados em um subgrupo envolvendo 23 crianças e seus respectivos pais, obteve-se diferença estatisticamente significante na comparação entre as respostas $(\mathrm{p}<0,029)$, na qual as crianças relataram mais episódios de cinetose em relação ao que foi percebido pelos pais. Acredita-se que a avaliação com um subgrupo de $\mathrm{n}$ maior tem demasiada relevância para os estudos sobre esta temática e com esta população. Esse subgrupo foi constituído por 23 pais e/ou responsáveis de crianças participantes do estudo, que responderam ao mesmo questionário utilizado (MSSQ). Durante a aplicação do protocolo, foram avaliadas as percepções dos adultos sobre a suscetibilidade à cinetose das crianças.

No momento em que se aplicava o protocolo, queixas como dificuldade de aprendizagem e de equilíbrio foram relatadas pelas crianças e/ou percebidas pelos avaliadores. Estudos apontam que as alterações vestibulares podem prejudicar o desenvolvimento motor, dificultando o contato da criança com o meio ambiente e a aquisição e desenvolvimento da linguagem ${ }^{21}$. Percebe-se também influência na aprendizagem e na habilidade de comunicação, tais como dificuldades de fala, leitura, escrita, soletração e cálculos matemáticos na criança em idade escolar ${ }^{22}$.

A manutenção da estabilidade postural durante a leitura pode ser uma tarefa mais complexa para as crianças com alteração vestibular, visto que o processo de leitura engloba muitas habilidades, como percepção, movimentos oculares e noções linguísticas e semânticas ${ }^{23,24}$. A dificuldade de aprendizagem pode ser caracterizada como a interação de uma série de fatores que resultam no baixo rendimento frente à situação de aprendizagem ${ }^{25}$.

No presente estudo, as queixas e/ou dificuldades de aprendizagem apareceram em pequena parte da amostra, o que não permitiu uma análise específica desta variável. No entanto, a literatura científica traz informações importantes sobre a relação entre equilíbrio e aprendizagem, e as implicações que as alterações vestibulares podem ter no processo de alfabetização e desenvolvimento da linguagem oral e escrita. Estudos que aprofundem ambas as temáticas e relacionem tais variáveis são necessários.

O questionário MSSQ mostrou-se um instrumento eficiente para a avaliação da suscetibilidade à cinetose na população infantil. $O$ instrumento possui fácil e rápida aplicação e compreensão, além de ter sido bem adaptado para a língua portuguesa e para a cultura brasileira. Portanto, recomenda-se a utilização deste protocolo para a realização de novas pesquisas que abordem as alterações vestibulares na infância.

\section{CONCLUSÃO}

A suscetibilidade à cinetose foi observada em 89,7\% da amostra, sendo as crianças do sexo feminino mais suscetíveis. Crianças com onze anos de idade apresentaram maior média no MSA. Houve diferença nas respostas relatadas pelas crianças e pelos pais avaliados a respeito da suscetibilidade à cinetose dessas crianças.

Sendo assim, justifica-se a importância de um olhar mais atento dos profissionais de saúde ao equilíbrio infantil, visto as diversas repercussões das alterações vestibulares no desenvolvimento. A avaliação e a reabilitação vestibular na infância se fazem necessárias na prática fonoaudiológica e nas demais áreas da saúde atuantes nesta temática. Acredita-se que crianças com cinetose podem ter inúmeros benefícios com a reabilitação vestibular, realizada pelos fonoaudiólogos, fisioterapeutas e fisiatras.

\section{REFERÊNCIAS}

1. Golding JF. Motion sickness susceptibility. Auton Neurosci. 2006;129:67-76. doi: 10.1016/j.autneu.2006.07.019.

2. Franco ES, Caetanelli EB. Avaliação vestibular em crianças sem queixas auditivas e vestibulares, por meio da vectoeletronistagmografia computadorizada. Int Arch Otorhinolaryngol [Internet]. 2006 [cited 2021 Aug 13];10(1):46-54. Available from: https://bit.ly/37G2Vaq

3. França SR, Perez, MLVD, Scharlach RC, Branco-Barreiro FCA. Susceptibilidade à cinetose em escolares. RECES. 2015;7(2):47-50. doi: 10.17921/2176-9524.2015v7n2p47-50.

4. Golding JF, Kadzere PN, Gresty MA. Motion sickness susceptibility fluctuates through the menstrual cycle. Aviat Space Environ Med [Internet]. 2005 [cited 2021 Aug 13];76(10):970-3. Available from: https://bit.ly/3mOhRbK 
5. França SR, Branco-Barreiro FCA. Susceptibilidade à cinetose no idoso com doença vestibular. RECES [Internet]. 2013 [cited 2021 Aug 13];5(1):30-5. Available from: https://bit.ly/37FrMet

6. Said TS. Prevalência de queixas de sintomas vestibulares em crianças [dissertation on the Internet]. São Paulo: Pontifícia Universidade Católica de São Paulo; 2012 [cited 2021 Aug 13]. Available from: https://bit.ly/3xEpXcg

7. Russell G, Abu-Arafeh I. Paroxysmal vertigo in children: an epidemiological study. Int J Pediatr Otorhinolaryngol, 1999;49(Suppl 1):S105-7. doi: 10.1016/s0165-5876(99)00143-3.

8. Bittar RSM, Pedalini MEB, Medeiros IRT, Bottino MA, Bento RF. Reabilitação vestibular na criança: estudo preliminar. Rev Bras Otorrinolaringol. 2002;68(4):496-9.

9. Silva BMP, Didoné DD, Sleifer P. Potencial evocado miogênico vestibular cervical em crianças e adolescentes sem queixas vestibulares. Audiol Commun Res. 2017;22:e1885. doi: 10.1590/2317-6431-2017-1885.

10. Lipson S, Wang A, Corcoran M, Zhou G, Brodsky JR. Severe motion sickness in infants and children. Eur J Paediatr Neurol. 2020;28:176-9. doi: 10.1016/j.ejpn.2020.06.010.

11. Huppert D, Grill E, Brandt T. Survey of motion sickness susceptibility in children and adolescents aged 3 months to 18 years. J Neurol. 2019;266(Suppl 1):65-73. doi: 10.1007/ s00415-019-09333-w.

12. Zhang LL, Wang JQ, Qi RR, Pan LL, Li M, Cai YL. Motion Sickness: Current Knowledge and Recent Advance. CNS Neurosci Ther. 2016;22(1):15-24. doi: 10.1111/cns.12468.

13. Takahashi M, Toriyabe I, Takei Y, Kanzaki J. Study on experimental motion sickness in children. Acta Otolaryngol. 1994;114(3):231-7. doi: 10.3109/00016489409126049. PMID: 8073856.

14. Dobie T, McBride D, Dobie T Jr, May J. The effects of age and sex on susceptibility to motion sickness. Aviat Space Environ Med. 2001;72(1):13-20.

15. Reason JT, Brand JJ. Motion sickness. Oxford: Academic Press; 1975.
16. Golding JF. Predicting individual differences in motion sickness susceptibility by questionnaire. Pers Individ Dif. 2006;41(2):237-48. doi: 10.1016/j.paid.2006.01.012.

17. Mariotto LDF. Avaliação vestibular em adolescentes com cinetose [dissertation on the Internet]. Botucatu: Universidade Estadual Paulista; 2007 [cited 2021 Aug 13]. Available from: https://bit.ly/3iGkRrE

18. Chang CH, Pan WW, Tseng LY, Stoffregen, TA. Postural activity and motion sickness during video game play in children and adults. Exp Brain Res. 2012;217:299-309. doi: 10.1007/ s00221-011-2993-4.

19. Henriques IF, Oliveira DWD, Oliveira-Ferreira F, Andrade PMO. Motion sickness prevalence in school children. Eur J Pediatr. 2014;173:1473-82. doi: 10.1007/s00431-014-2351-1.

20. Kennedy RS, Lanham DS, Massey CJ, Drexler JM, Lilienthal MG. Gender differences in simulator sickness incidence: implications for military virtual reality systems. Safe J. 1995;25(1):69-76.

21. Formigoni LG, Medeiros IRT, Santoro PP, Bittar RSM, Bottino MA. Avaliação clínica das vestibulopatias na infância. Braz J Otorhinolaryngol. 1999;65(1):78-82. doi: 10.1590/ S0034-72992002000400007.

22. Franco ES, Panhoca I. Sintomas vestibulares em crianças com queixa de dificuldades escolares. Rev Soc Bras Fonoaudiol. 2008;13(4):362-8. doi: 10.1590/S1516-80342008000400011.

23. Legrand A, Bui-Quoc E, Doré-Mazars K, Lemoine C, Gérard CL, Bucci MP. Effect of a dual task on postural control in dyslexic children. PLoS One. 2012;7(4):e35301. doi: 10.1371/journal. pone.0035301.

24. Tomaz A, Ganança MM, Garcia AP, Kessler N, Caovilla HH. Controle postural de escolares com baixo rendimento escolar. Braz J Otorhinolaryngol. 2014;80(2):105-10. doi: 10.5935/18088694.20140024.

25. Rosa Neto F, Amaro KN, Prestes DB, Arab C. O esquema corporal de crianças com dificuldade de aprendizagem. Psicol Esc Educ. 2011;15(1):15-22. doi: 10.1590/S1413-85572011000100002. 